\title{
Correlation between oral health-related quality of life (OHQoL) and oral disorders in a Turkish patient population
}

\author{
Fatma Caglayan ${ }^{1}$, Oguzhan Altun ${ }^{1}$, Ozkan Miloglu ${ }^{1}$, Muhammed-Dursun Kaya ${ }^{2}$, Ahmet-Berhan Yilmaz ${ }^{3}$
}

\author{
${ }^{1}$ Researcher, Department of Oral Diagnosis and Radiology, Faculty of Dentistry, Ataturk University, Erzurum/Turkey \\ ${ }^{2}$ Assistant Professor, Department of Vocational Collage at Erzurum, Ataturk University, Erzurum/Turkey \\ ${ }^{3}$ Professor, Department of Oral Diagnosis and Radiology, Faculty of Dentistry, Ataturk University, Erzurum/Turkey
}

Correspondence:

Department of Oral Diagnosis and Radiology,

Faculty of Dentistry, Ataturk University,

25240 Erzurum / TURKEY

facagla@gmail.com

Received: 04/11/2008 Accepted: 10/03/2009

\begin{abstract}
Caglayan F, Altun O, Miloglu O, Kaya MD, Yilmaz AB. Correlation between oral health-related quality of life $(\mathrm{OHQoL})$ and oral disorders in a Turkish patient population. Med Oral Patol Oral Cir Bucal. 2009 Nov 1;14 (11):e573-8.

http://www.medicinaoral.com/medoralfree01/v14i11/medoralv14i11p573.pdf
\end{abstract}

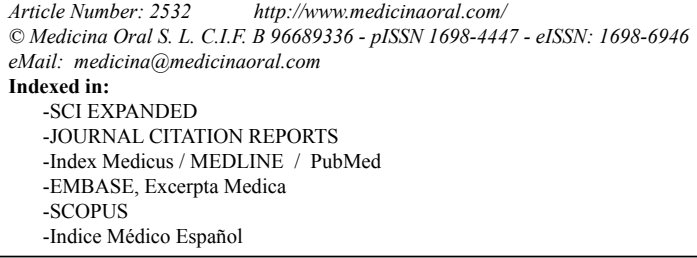

\begin{abstract}
Objectives: The purpose of the present study is to determine the nature of the complaints that bring patients to our clinic and to what degree these complaints affect their quality of life $(\mathrm{QoL})$. We also aimed to determine any associations between gender, education or harmful habits and each patient's oral health-related quality of life (OHQoL). Methods: A total of 1090 patients, consisting of 651 females (59.7\%) and 439 males (40.3 \%), were included in this study. Of these patients, 220 constituted healthy controls. Two patient-centered outcome measures, the 14 item OHIP-14 and the 16 item OHQoL-UK measures were used. Results: Most of the patients presented with toothache and caries (50.1\%), $11.2 \%$ had suffered tooth loss and had denture needs, $9.2 \%$ had periodontal problems, $1.8 \%$ had temporomandibular joint (TMJ) disorders, $3.8 \%$ had buried third molars, $2.4 \%$ had orthodontic and aesthetic disorders, $1.3 \%$ had suffered injury due to trauma, and $20.2 \%$ came only for control checkups. OHQoL was best in the control group and the worst in patients who had suffered trauma. In addition, we noted correlations between gender, education and harmful habits, and that of the patient's oral health-related quality of life. Conclusion: According to our results, OHQoL is associated with the oral complaints of patients. Furthermore, OHQoL may not only be associated with the oral health status of patients, but factors such as gender, education and harmful habits may also play a role.
\end{abstract}

Key words: Oral health, quality of life. 


\section{Introduction}

Patient-centered approaches have been receiving increasingly more attention in recent years. It is important to determine the nature of the complaints that cause patients to seek treatment and to what degree these affect patients' quality of life (QoL). There is an increasing recognition that oral health has a significant impact on not only physical, but also social and psychological well-being. As in general medicine, perceptions of dental patients are also important in the assessment of treatment need, in planning of appropriate therapy and in clinical outcome. The patient-centered outcome measures may therefore also be utilized in oral medicine. While the majority of oral diseases are not fatal, they can give rise to significant morbidity, resulting in physical, social and psychological consequences which affect patients' QoL (1).

Interest in the idea of quality of life is growing rapidly. More than 1000 new articles are indexed each year under this heading (2). QoL is affected by oral health in some way in the majority of people (3). A variety of patient-centered outcome measures termed 'oral health related quality of life measures' (OHQoL) have been developed to assess the extent to which oral health problems affect not only physical functioning and pain, but broader constructs such as psycho-social functioning and life satisfaction (1).

A number of OHQoL measures have been developed and are presently being evaluated. Eleven $\mathrm{OHQoL}$ measures were reviewed at an international meeting held at the University of North Carolina in 1996 (4). It was mentioned that two measures which had received particular attention were the Oral Health Impact Profile (OHIP-14) and the UK Oral Health Related Quality of Life $\left(\mathrm{OHQoL}-\mathrm{UK}^{\odot}\right)$ questionnaires $(5,6)$. These measures are based on two conceptually distinct models of oral health. The OHIP-14 consists of self-reported measurements of the adverse impacts of oral conditions on daily life. Originally developed in Australia, it is based on a conceptual model of oral health that uses the World Health Organization (WHO) International Classification of Impairments, Disabilities and Handicaps framework (7). The original 49-item questionnaire has been shortened to 14 items by Slade and has allowed use of a validated index of the impact of oral health (5). Since its development, the OHIP-14 is preferred to the OHIP-49 by a number of researchers due to its practicality.

OHQoL-UK, recently developed in the United Kingdom, is based on the WHO model of "structure-function-ability-participation", which incorporates both negative and positive influences on health (8).

The purpose of the present study is to determine which complaints cause patients to come to our clinic and to what degree these complaints affect their QoL. Furthermore, we aimed to determine if there was a possible association between gender, education, harmful habits and their oral health QoL.

\section{Materials and Methods}

Study population

This clinical-based descriptive study was carried out in the Oral Diagnosis and Radiology Department of the Faculty of Dentistry, Ataturk University. Consecutive 1090 patients were included the study. 220 of these constituted healthy controls. Patients less than 18 years old and patients who could not give adequate data were not included the study.

The examination of patients and application of questionnaires were carried out by three researchers. Firstly, each patient's name, surname, age, gender, place of birth and educational status were recorded, and a medical history was taken. Any systemic disease or drug use was noted. Clinical and radiographic examinations were performed. After intra-oral examination, dental outpatients were asked to complete patient-centered outcome measures. For standardization of the study, the first researcher trained the other two researchers in the clinical assessment and implementation of the questionnaires. All researchers agreed upon which questionnaires would be included or excluded in the study. The questionnaires were implemented in a face to face interview.

\section{Data Collection}

Two patient-centered outcome measures, the 14 item OHIP-14 and the 16 item OHQoL measure (OHQoLUK) were used in this study. The questionnaires were translated into Turkish, in accordance with cross-cultural adaptation guidelines, to produce a Turkish version of the OHIP-14 and the OHQoL $(9,10)$. Both measures had been previously validated with Turkish dental outpatients (9).

Data Analysis

Scores were derived from both questionnaires by summing the responses to each of the individual questions within the measures. The questions for OHIP-14 were asked as "..................because of your teeth, mouth or denture?" For the OHIP-14, each item was scored: 'never'- score 0 , 'hardly ever'- score 1 , 'occasionally'- score 2, 'fairly often'- score 3, 'very often'- score 4. Higher scores indicate poorer oral health-related quality of life. The questions for OHQoL-UK were asked as "What effect does your oral health have on your................" For the OHQoL-UK, the response categories were 'very bad effect'-score 1, 'bad effect'score 2, 'no effect'- score 3, 'good effect'- score 4, 'very good effect'- score 5. Lower scores indicate poorer oral health-related quality of life. Thus, better OHQoL was indicated with lower scores in OHIP-14, and with higher scores in OHQoL-UK questionnaires.

The collected data were analyzed by SPSS 10.0 soft- 
ware program. The Mann-Whitney test was used to compare the OHQoL of females and males. KruskalWallis test was used to identify differences in OHQoL with patients' complaints, education, and harmful habits. The OHIP-14 and OHQoL-UK scores of groups are expressed as median (interquartile range). $\mathrm{p}<0.05$ was considered statistically significant.

\section{Results}

The demographic characteristics of the subjects, their educational status, their oral health complaints and the distributions of their habits (smoking and alcohol intake) are shown in Table 1. According to these 651 (59.7\%) females and 439 (40.3\%) males, a total of 1090 patients were included in the study. Mean age was $29.61 \pm 11.03$, and patients between age 18-27 (56.4\%) comprised a

Table 1. Demographic characteristics of subjects.

\begin{tabular}{|l|c|}
\hline & n (\%) \\
\hline $\begin{array}{l}\text { Gender } \\
\text { Male }\end{array}$ & $651(59.7)$ \\
\hline Age & $439(40.3)$ \\
\hline $18-27$ & \\
\hline $28-37$ & $618(56.4)$ \\
\hline $38-47$ & $234(21.4)$ \\
\hline $48-57$ & $140(12.7)$ \\
\hline $58-67$ & $72(7.0)$ \\
\hline Education & $26(2.5)$ \\
\hline None & $22(2.0)$ \\
\hline Primary & $286(26.2)$ \\
\hline High school & $290(26.6)$ \\
\hline University & $480(44.1)$ \\
\hline Master & $12(1.1)$ \\
\hline Complaints of patients & \\
\hline Caries, toothache & $546(50.1)$ \\
\hline Periodontal problems & $100(9.2)$ \\
\hline Toothless, dentures & $122(11.2)$ \\
\hline TMJ problems & $20(1.8)$ \\
\hline Third molars & $42(3.8)$ \\
\hline Trauma & $14(1.3)$ \\
\hline Orthodontic/aesthetic & $26(2.4)$ \\
\hline Control & $220(20.2)$ \\
\hline Habits & $10(0.9)$ \\
\hline No habit & $846(77.6)$ \\
\hline Smoking & $228(20.9)$ \\
\hline Alcohol intake & $6(0.6)$ \\
\hline Both smoking and alcohol & \\
\hline & \\
\hline & \\
\hline
\end{tabular}

large part of it. Most of the patients generally came to the clinic with the complaint of caries and toothache (50.1\%), $11.2 \%$ tooth loss and denture need, $9.2 \%$ periodontal complaints, $1.8 \%$ TMJ complaints, $3.8 \%$ buried third molars, $2.4 \%$ orthodontic and aesthetic defects, $1.3 \%$ trauma, and $20.2 \%$ for only control. While $77.6 \%$ of the patients have no harmful habits, $20.9 \%$ of them were smokers, $0.6 \%$ was drinking alcohol and $0.9 \%$ was both smokers and drinking alcohol.

The median scores of patients' answers to OHIP-14 and OHQoL-UK questionnaires are shown in Figure 1 and Figure 2. The main difference between OHQoL-UK

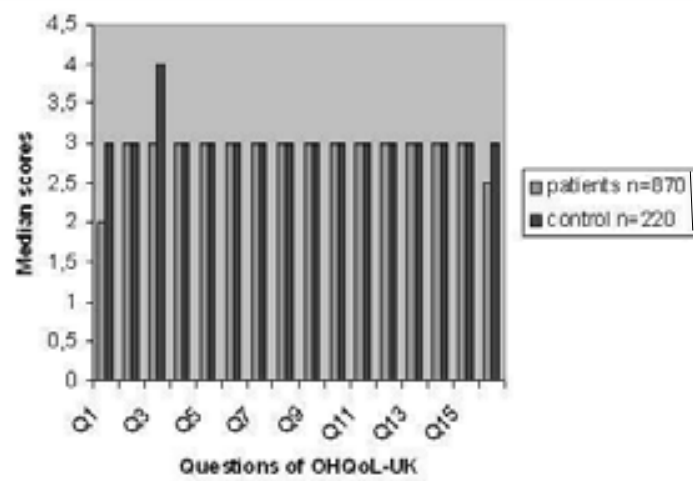

Fig. 1. The questions and median scores of OHQoL-UK. (Q1: Eating enjoyment of food, Q2: Appearance, Q3: Speech, Q4: General health, Q5: Ability to relax, Q6: Social life, Q7: Romance, Q8: Smiling/laughing, Q9: Confidence, Q10: Carefree manner, Q11: Mood, Q12: Work/usual duties, Q13: Finances, Q14: Personality, Q15: Comfort, Q16: Breath odor).

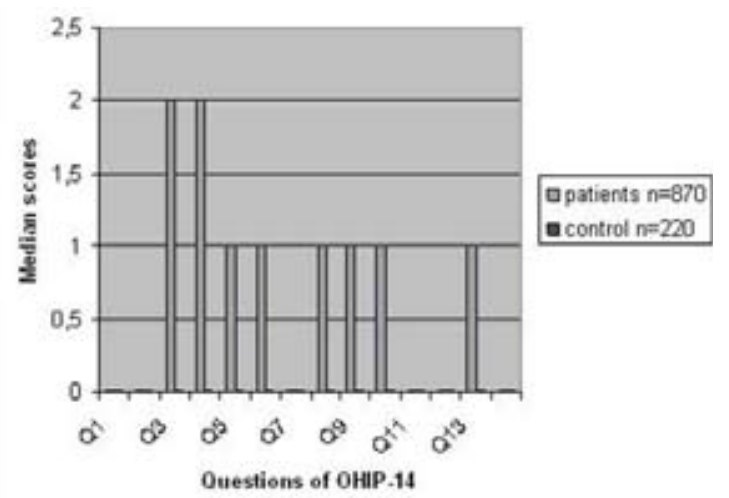

Fig. 2. The questions and median scores of OHIP-14. (Q1: Trouble pronouncing words, Q2: Taste worse, Q3: Painful aching, Q4: Uncomfortable to eat, Q5: Self-conscious, Q6: Tense, Q7: Diet unsatisfactory, Q8: Interrupt meals, Q9: Difficult to relax, Q10: Embarrassed, Q11: Irritable, Q12: Difficult doing your usual jobs, Q13: Life less satisfying, Q14: Totally unable to function). 
Table 2. OHQoL-UK and OHIP-14 scores according to gender, complaint, education and habits.

\begin{tabular}{|c|c|c|c|c|c|}
\hline & n & $\begin{array}{c}\text { OHQOL- } \\
\text { UK } \\
\text { Median } \\
\text { (interquar- } \\
\text { tile range) }\end{array}$ & P value & $\begin{array}{c}\text { OHIP-14 } \\
\text { Median } \\
\text { (interquartile } \\
\text { range) }\end{array}$ & Pvalue \\
\hline \multicolumn{6}{|l|}{ Gender } \\
\hline Female & 651 & $2.87(0.81)$ & 0.292 & $0.86(1.00)$ & $0.010^{*}$ \\
\hline Male & 439 & $2.87(1.00)$ & & $0.64(1.00)$ & \\
\hline \multicolumn{6}{|l|}{ Complaint } \\
\hline Caries, toothache & 546 & $2.81(0.78)$ & $0.000 * *$ & $0.93(1.07)$ & $0.000 * *$ \\
\hline Periodontal problems & 100 & $2.91(0.69)$ & & $0.68(1.07)$ & \\
\hline Toothless, dentures & 122 & $2.69(0.50)$ & & $0.86(0.86)$ & \\
\hline TMJ problems & 20 & $2.87(1.19)$ & & $1.11(0.71)$ & \\
\hline Third molars & 42 & $2.75(1.14)$ & & $0.93(0.53)$ & \\
\hline Trauma & 14 & $2.44(0.89)$ & & $1.25(1.71)$ & \\
\hline Orthodontic/aesthetic & 26 & $2.81(0.50)$ & & $0.43(0.61)$ & \\
\hline Control & 220 & $3.18(1.12)$ & & $0.21(0.64)$ & \\
\hline \multicolumn{6}{|l|}{ Education } \\
\hline None & 22 & $2.68(0.62)$ & $0.000 * *$ & $0.93(0.58)$ & $0.000 * *$ \\
\hline Primary & 286 & $2.81(0.56)$ & & $0.93(1.14)$ & \\
\hline High school & 290 & $2.87(0.75)$ & & $0.71(1.00)$ & \\
\hline University & 480 & $2.94(1.19)$ & & $0.64(0.86)$ & \\
\hline Master & 12 & $2.97(1.58)$ & & $0.61(0.41)$ & \\
\hline \multicolumn{6}{|l|}{ Harmful habits } \\
\hline No habit & 846 & $2.94(0.94)$ & $0.001 * *$ & $0.71(0.93)$ & $0.000 * *$ \\
\hline Smoking & 228 & $2.75(0.62)$ & & $0.93(1.00)$ & \\
\hline Alcohol & 6 & $2.87(0.62)$ & & $0.57(2.14)$ & \\
\hline Smoking and alcohol & 10 & $2.75(0.50)$ & & $0.71(0.66)$ & \\
\hline
\end{tabular}

scores of patients and controls were found in Q1, Q4 and Q16, representing eating, general health and breathe odor respectively. And the main difference between the OHIP-14 scores of patients and controls were found in Q3, Q4 representing painful aching and uncomfortable to eat respectively. That is, eating was the common problem affected by oral complaints in both questionnaires.

Table 2 illustrates the scores of OHIP-14 and OHQoLUK with respect to the patients'complaints, gender, education and harmful habits. There was no statistically significant association between gender and OHQoL-UK $(p>0.05)$. The median OHQoL-UK score of females was 2.87 (0.81) and males 2.87 (1.00). However, OHIP14 scores of females were higher than those of males $(\mathrm{p}<0.05)$. The median OHIP-14 score of females was 0.86 (1.00), males 0.64 (1.00).

There was a significant difference in OHQoL-UK $(p<0.001)$ and OHIP-14 $(p<0.001)$ scores of controls and patients according to complaints. The median OHQoLUK score of patients was 2.81(0.75) and controls 3.18 (1.12). The median OHIP-14 score of patients was 0.86 (0.93) and controls $0.21(0.64)$. OHIP-14 scores were highest and OHQoL-UK scores were lowest in patients who came with trauma. OHIP-14 scores were lowest and OHQoL-UK scores were highest in patients who came for checkups.

There was a significant association between education and OHQoL-UK $(\mathrm{P}<0.001)$ and OHIP-14 $(\mathrm{P}<0.001)$. 
When education level was promoted, OHQoL also became better. There was also a significant association between harmful habits and QHQoL. Patients who had no harmful habits had higher QHQoL-UK $(\mathrm{P}<0.01)$ scores and lower OHIP-14 scores $(\mathrm{P}<0.001)$ than patients who were smokers or drinking alcohol.

\section{Discussion}

The impact of health on the quality of life (QoL) has received increased attention in both medicine and dentistry. Locker and Gibson defined positive health as the absence of negative health states, positively worded items, positive outcomes of oral health, a set of psychological and social attributes, and positive outcomes of chronic conditions such as oro- and craniofacial differences (11). Mc Grath et al. claimed that positive and negative health states and experiences are distinct, in that "the absence of a negative does not necessarily imply a positive and a positive state can coexist with a negative state" (12). The QHQoL-UK attempts to asses both positive and negative effects of oral health, while the OHIP-14 assesses only negative effects of oral health (11). So this is a limitation for OHIP-14 in capturing the global conception of health and well-being.

Kushnir et al. mentioned that oral health status was closely associated with QoL, and that a problem in oral health might seriously decrease a patient's QoL (13). On the other hand, Gregory et al. mentioned that quality of life could be variable, according to patient perceptions (14). Therefore, the associations between quality of life and clinical status can be weak or non-existent. In the present study, we tried to handle the issue from the patient's perspective and we used each patient's first complaint which made them to come to our clinic. First of all, we determined the nature of their complaints and then if there was an association between these complaints and their OHQoL. Unfortunately, in our society, people do not tend to care about their oral and dental health problems, as long as these problems do not result in aching or are otherwise disturbing them. While the rate of the patients who came to the clinic for only checkups without any complaints was $20.2 \%$, the rate of the patients who came with a complaint was $79.8 \%$. Quality of life was the highest in patients who came to our clinic for only dental control. OHIP-14 scores were the lowest but OHQoL-UK scores were the highest in these patients, representing a better OHQoL.

Tooth decay and toothache are the foremost complaints among patients, with range of $50.1 \%$. According to our results, OHQoL of patients with caries and toothache was poorer than control group. Similarly Ng et al. found out that QoL of patients without toothache was better than that of patients with toothache (2).

In this study, the rate of patients who were referred to our clinic because of periodontal problems was $9.2 \%$.
$\mathrm{Ng}$ et al. found that periodontal problems affect the QoL in a negative way (2). We have found OHIP-14 values high and OHQoL-UK values low in patients who have periodontal complaints compared to the patients who refer to our clinic for checkups, indicating a lower QoL.

There are no studies reported in the literature concerning the impact of maxillofacial trauma on OHQoL in adult patients, although a high prevalence of traumatic injuries in childhood and adolescence has been described in the literature. Cortes et al. found that children with untreated dental fracture of permanent teeth had more impacts on their daily living than did children without any dental trauma (15). According to our results, trauma was the complaint that had the most negative effect on QoL. While the OHIP-14 values were the highest in patients who came with trauma, the OHQoLUK values were the lowest. OHIP-14 may not be useful to asses the impact of acute and severe trauma on OHQoL indeed. Because, OHIP-14 assess the quality of life in terms of frequency (from never to very often) and traumatic injuries, are acute and severe events that should not be properly assessed by this sort of instruments unless subjects confound frequency with severity. But as we mentioned above, we did not include patients who gave inadequate data. The trauma patients included in our study were the ones who underwent maxillofacial trauma previously and had not treated suitable. Gianetti et al. found out that dental avulsion impaired OHQoL in patients under 18 years old by using OHIP-14 (16).

Tooth loss affects general health and well being, in addition to oral health related QoL. Steele reported that QoL is increasingly affected as the number of missing teeth increases (3). We have also found that OHIP-14 values are higher and OHQoL-UK values are lower in patients who have complaints of tooth loss than are those of patients who were referred to our clinic for checkups, indicating a poorer OHQoL associated with tooth loss.

There are indications that many patients suffering from TMJ disorders may also show a reduced OHQoL (17). We have also found low OHQoL-UK values and high OHIP14 values in patients with TMJ disorders in comparison with patients who came for checkups, again representing a poorer $\mathrm{OHQoL}$ in TMJ patients. Malocclusion also has negative effects on QoL (18). In the present study, we have also found that $\mathrm{OHQOL}$ is lower in patients who were referred to us for orthodontic and aesthetic disorders. According to our results, OHQoL of patients with buried third molars and pericoronitis was also reduced, in agreement with a study by Mc Grath et al., who also pointed out that pericoronitis impaired the QoL (19).

According to our results, there was no significant difference between the OHQoL-UK scores of males and females. However, OHIP-14 scores of females were higher than were those of males. Fernandes et al. found no significant difference between the OHIP-14 scores of 
males and females (20). However, Steele et al. found the OHIP-14 scores of females to be higher in the United Kingdom and Australia, in agreement with our study (3). That is to say, QoL of females appeared to be more susceptible to disruption by oral disorders.

Fernandes et al. found that OHIP-14 scores of patients who were smokers to be higher, similar to the results of the present study (20). The negative effect of smoking to OHQoL is probably due to the harmful effects of smoking on oral tissues. Fernandes et al. found OHQoL to be better in patients who drank alcohol in the same study (20). We also found OHQoL of patients who drank alcohol to be better than patients who both drank alcohol and smoked.

In this study, we have also found OHQoL-UK values to be higher in patients who have low OHIP-14 scores, in agreement with the literature. While low OHIP-14 values indicate good QoL, low OHQoL-UK values indicate poor QoL $(1,9)$ and vice versa. This is the first study that has been carried out that considers patient complaints and the effect of these complaints on their QoL in our society. The conclusion we have reached is that OHQoL is poorer in patients who have complaints of oral disorders, and that this is associated with oral health status. Furthermore, OHQoL is associated with gender, educational status, smoking and alcohol intake.

\section{References}

1. Hegarty AM, McGrath C, Hodgson TA, Porter SR. Patient-centred outcome measures in oral medicine: are they valid and reliable? Int $\mathrm{J}$ Oral Maxillofac Surg. 2002;31:670-4.

2. Ng SK, Leung WK. Oral health-related quality of life and periodontal status. Community Dent Oral Epidemiol. 2006;34:114-22.

3. Steele JG, Sanders AE, Slade GD, Allen PF, Lahti S, Nuttall N, et al. How do age and tooth loss affect oral health impacts and quality of life? A study comparing two national samples. Community Dent Oral Epidemiol. 2004;32:107-14.

4. Slade GD, Strauss RP, Atchison KA, Kressin NR, Locker D, Reisine ST. Conference summary: assessing oral health outcomes-measuring health status and quality of life. Community Dent Health. 1998;15:3-7.

5. Slade GD. Derivation and validation of a short-form oral health impact profile. Community Dent Oral Epidemiol. 1997;25:284-90.

6. McGrath C, Bedi R. An evaluation of a new measure of oral health related quality of life--OHQoL-UK(W). Community Dent Health. 2001;18:138-43.

7. World Health Organization. The International Classification of Impairments, Disabilities and Handicaps (ICIDH) WHO, Geneva; 1980 .

8. World Health Organization. International Classification of Functioning, Disability and Health. Geneva; 2001.

9. Mumcu G, Inanc N, Ergun T, Ikiz K, Gunes M, Islek U, et al. Oral health related quality of life is affected by disease activity in Behçet's disease. Oral Dis. 2006;12:145-51.

10. Beaton DE, Bombardier C, Guillemin F, Ferraz MB. Guidelines for the process of cross-cultural adaptation of self-report measures. Spine (Phila Pa 1976). 2000;25:3186-91.

11. Locker D, Gibson B. The concept of positive health: a review and commentary on its application in oral health research. Community Dent Oral Epidemiol. 2006;34:161-73.

12. McGrath $\mathrm{C}$, Broder H, Wilson-Genderson M. Assessing the impact of oral health on the life quality of children: implica- tions for research and practice. Community Dent Oral Epidemiol. 2004;32:81-5.

13. Kushnir D, Zusman SP, Robinson PG. Validation of a Hebrew version of the Oral Health Impact Profile 14. J Public Health Dent. 2004;64:71-5.

14. Gregory J, Gibson B, Robinson PG. Variation and change in the meaning of oral health related quality of life: a 'grounded' systems approach. Soc Sci Med. 2005;60:1859-68.

15. Cortes MI, Marcenes W, Sheiham A. Impact of traumatic injuries to the permanent teeth on the oral health-related quality of life in 12-14-year-old children. Community Dent Oral Epidemiol. 2002;30:193-8.

16. Giannetti L, Murri A, Vecci F, Gatto R. Dental avulsion: therapeutic protocols and oral health-related quality of life. Eur J Paediatr Dent. 2007;8:69-75.

17. Türp JC, Motschall E, Schindler HJ, Heydecke G. In patients with temporomandibular disorders, do particular interventions influence oral health-related quality of life? A qualitative systematic review of the literature. Clin Oral Implants Res. 2007;18 Suppl 3:127-37.

18 Zhang M, McGrath C, Hägg U. The impact of malocclusion and its treatment on quality of life: a literature review. Int J Paediatr Dent. 2006;16:381-7.

19. McGrath C, Comfort MB, Lo EC, Luo Y. Can third molar surgery improve quality of life? A 6-month cohort study. J Oral Maxillofac Surg. 2003;61:759-63.

20. Fernandes MJ, Ruta DA, Ogden GR, Pitts NB, Ogston SA. Assessing oral health-related quality of life in general dental practice in Scotland: validation of the OHIP-14. Community Dent Oral Epidemiol. 2006;34:53-62. 